Voix et Images

voixetimages

\title{
Dans le ventre du récit (lecture de Gérard Bessette)
}

\section{Louis Hamelin}

Volume 20, numéro 2 (59), hiver 1995

Archéologie du littéraire au Québec

URI : https://id.erudit.org/iderudit/201173ar

DOI : https://doi.org/10.7202/201173ar

Aller au sommaire du numéro

\section{Éditeur(s)}

Université du Québec à Montréal

\section{ISSN}

0318-9201 (imprimé)

1705-933X (numérique)

Découvrir la revue

\section{Citer cet article}

Hamelin, L. (1995). Dans le ventre du récit (lecture de Gérard Bessette). Voix et Images, 20(2), 440-450. https://doi.org/10.7202/201173ar d'utilisation que vous pouvez consulter en ligne.

https://apropos.erudit.org/fr/usagers/politique-dutilisation/ 


\title{
Dans le ventre du récit (lecture de Gérard Bessette)
}

\author{
Louis Hamelin
}

Scène originaire: dans la chambre voisine de'la mienne, au soussol de la maison familiale, mon frère lit Le Libraire. Par la porte entrebâillée, je peux voir la souple couverture de carton jaune de l'édition de poche du Cercle du livre de France (qui ne paie pas de mine). Des bruits suspects me parviennent, alimentent mon imagination. Je perçois des éclats de rire étouffés, décide que le livre doit appartenir au genre comique, comme La Foire aux cancres, ce recueil de perles estudiantines dont nous nous repaissions, plus jeunes, sur la recommandation d'un oncle missionnaire qui, à la page idoine, nous mettait par ailleurs en garde: "Certains passages ne sont pas pour les oreilles pies. "Quant au professeur Bessette, son nom, déchiffré à la hâte au cours d'une incursion clandestine, me fait l'effet d'une bête curieuse. Je serais bien embêté de lui attribuer une nationalité ou l'autre. Pour moi, la littérature québécoise existe encore très peu, et le nom de Gérard Bessette me renvoie à un néant, à mon innocence dont je ne vais émerger que bien plus tard, une fois parvenu à l'université et prêt, avec mes yeux de lecteur tout neufs, à affronter les mânes du tout-puissant Père-Pédagogue-Ex-Écrivain-en-Résidence.

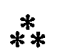

On a parlé, à propos du Libraire, de premier roman existentialiste québécois. La relative neutralité psychologique de Jodoin, ce cynisme qui semble aller de soi, rappellent peut-être en effet l'indifférence de Meurseault, de même que le style adopté par Bessette, dénué de passion, peut faire penser à la fameuse écriture blanche. Mais derrière l'attitude de ce narrateur, sous la netteté de sa prose et des thèmes apparents, se profile pourtant quelque chose qui n'a rien à voir (littéralement) avec une recherche formelle et philosophique importée d'Europe. Tout se passe comme si l'écriture blanche, au lieu de tra- 
vailler ici à dévoiler l'absurdité de la condition humaine, servait plutôt à tenir à distance, en respect, une imagerie dont la récurrence, dans la suite de l'œuvre, accusera rétrospectivement l'impeccable détachement de ce libraire. Si l'Absurde est présent dans ce livre, c'est d'une manière plus viscérale que raisonnée. L'aventure de Jodoin ne débouche sur aucune leçon, aucune prise de conscience qui appellerait, comme nécessaire corollaire, la révolte ou la liberté. Elle aura à peu près les répercussions d'un simple dérangement un peu prolongé des habitudes, un ennui d'ordre physiologique bien plus que métaphysique. À l'esprit coulant du héros, qui lui évite toute confrontation directe, répond ce corps sarcastique qui, par sa force d'inertie, devient la véritable expression du refus dans le texte. Et Jodoin n'est que relativement étranger au petit monde de la Librairie Léon puisque, dans l'antre obscur qui s'ouvre au fond de l'arrière-boutique, comme une extension de sa propre psyché, dorment les livres refoulés.

Le placard de $\mathrm{M}$. Chicoine, réduit d'accès limité où sont gardés les volumes mis à l'index, est appelé capharnaüm, même si nul désordre apparent ne caractérise le lieu, comme peut le constater Jodoin à sa première visite. On peut soupçonner Bessette, latiniste distingué, plus tard psycho-critique patenté et redoutable décortiqueur de mots, de s'être montré sensible à certains aspects graphiques ou phoniques, ou encore à la simple étymologie de ce capharnaüm: de cafourniau, "débarras", et de furnus, "four". Jodoin, à un ancien collègue qui lui demande s'il aime toujours les livres, répond que "les livres brûl(ent) moins longtemps que le charbon, mais que, faute d'autre combustible, il [lui arrive] de [s'en] servir" (p. 23). De plus, la dialectique de l'embarras et du débarras est centrale chez Bessette, où l'acte sexuel sera souvent présenté comme une façon de "se vider les couilles"'(Les Dires d'Omer Marin). Déjà, dans Le Libraire, le cheminement organique est au premier plan, et l'excrétion fait partie des préoccupations incontournables du narrateur. Dans le capharnaüm, on trouve de plus un cafard, ce dont l'auteur se montre tout à fait conscient dans le passage suivant:

- Vous avez dû rester surpris quand vous vous êtes aperçu que votre clef n'ouvrait pas le capharnaüm? m'a-t-il demandé. Les lèvres figées en un sourire cafard qui découvrait ses longues dents chevalines... (p. 61).

De l'arabe kafir, "mécréant, renégat", le mot désigne, dans son sens premier, une personne qui affecte l'apparence de la dévotion. Appliqué au cabinet des livres proscrits, le terme capharnaüm nous situe donc d'emblée au cœur de la problématique développée dans $L e$ Libraire. Entre l'hypocrite Chicoine et Jodoin le renégat, ce qui va se jouer à travers la médiation du curé, c'est l'épreuve de la Loi du Père, 
thème appelé à devenir, chez Bessette, le sujet obsédant par excellence. Dans Le Semestre, derrière le déguisement extrêmement mince d'Omer Marin, il livrera sous forme d'interrogation la confession suivante:

[...] chez moi ce penchant ce désir d'évasion-libération ne découle-t-il pas de ma précoce révolte inconsciente contre les enseignements de ma mère et de l'Église, laquelle (mère) se trouvait éloignée de moi par l'écran de sa foi fanatique envers l'Église-écran qui lui permettait de ravaler-négliger le père de ses enfants en faveur de son défunt père et du Père éternel?...) (p. 40)

Le vrai rival odipien serait donc, d'après ce schéma, le Père éternel et patriarcal ayant pris, dans le cour de la mère, la place de l'autre, simple ersatz biologique et bedeau de son métier en plus. La description du capharnaüm, avec son "globe laiteux" pendu au plafond (p. 44), suffirait peut-être alors à féminiser celui-ci si l'auteur n'en rajoutait pas, usant de procédés de déplacement qui établissent la relation du personnage à cet espace :

[...] quand j'y mis les pieds pour la première fois, j'avais un solide coup de scotch dans le nez. Je m'en envoyai incontinent un autre pour me donner du cour au ventre (p. 44).

Dans son livre intitulé Mes romans et moi, dont la première partie constitue un rudiment d'autobiographie, Bessette fera preuve plus tard, à travers les méandres de la remémoration, d'une sensibilité spatiale toute particulière. Il évoquera par exemple les "recoins intéressants" de la maison familiale, sa cave "mystérieuse et sombre" et, sous les combles, les deux immenses "chambres à débarras" où l'enfant fait de fréquents séjours. Dans sa préface au livre, Jacques Allard fait remarquer que cette maison, à cause de l'office rempli par le père, est à toutes fins utiles conçue comme une simple dépendance de l'église du village.

Lieu tabou, le capharnaüm l'est donc doublement: d'abord comme bibliothèque clandestine renfermant les livres à ne pas mettre entre toutes les mains; ensuite, par la position hautement métaphorique qu'il occupe dans la librairie (à la fois centrale et en retrait), et la charge sémantique dont l'écriture l'investit pour en faire le symbole de ce fantasme utérin autour duquel une bonne partie de l'œuvre postérieure de Bessette est destinée à graviter.

Il est frappant de constater le dédain souverain affiché par Jodoin à l'endroit des ouvres qu'abrite le placard. On ne le voit jamais lire. Accoudé au comptoir de la librairie, un bouquin ouvert devant lui, il préfère roupiller. Et pourtant, malgré qu'il s'en défende, il est évident 
qu'il a lu et que la chair est triste. Il ne se prive d'ailleurs pas de critiquer vertement l'Essai sur les mœurs et le sieur Voltaire lui-même. Mais sa relation au contenu du cagibi n'est pas dynamique. C'est le rapport passif d'un être repu à l'aliment assimilé. Le livre, lui-même contenant, devient dans la perspective de ce roman un simple contenu. À la fin, après avoir vidé nuitamment le placard de $M$. Chicoine, Jodoin précisera : "L'urgence [...] concernait uniquement l'évacuation des bouquins..." (p. 151).

Les habitudes de ce commis de librairie trahissent une évidente fixation liquide. L'absorption et l'excrétion sont les deux pôles d'un assouvissement passif et stö̈que. Coincé entre ses vingt bocks vespéraux et la porte des pissotières, Jodoin règle son existence entière sur une idée de cycle: ingurgitation-expulsion, circulation-élimination. Il évolue au centre d'un flux que son organisme contrôle, recréant le havre béni, le bain primordial d'où tout imprévu était banni. Le nom du sel digestif utilisé pour régulariser le processus dit tout: Safe-All.

Devant le capharnaüm, Jodoin adoptera donc un comportement ambivalent. Ses visites dans cette "chambre noire" lui répugnent: "ça [l']emmerde d'aller fouiller " là-dedans " (p. 63). Mais du même coup, à son corps défendant, il se retrouve paré d'un rôle positif: celui d'introducteur, d'initiateur à qui revient de forcer un secret. Le récit s'attarde non sans insistance sur le sort de la clef qu'on lui confie, comme pour rendre encore plus limpide la métaphore sexuelle. D'une part, le vieux pédagogue libidineux du Semestre se profile déjà dans ce commis de librairie qui, par sympathie et une bonne dose d'identification, refile à un collégien le livre qui va provoquer le scandale et sa propre expulsion en douce du village; d'autre part, Salaloudi-le-voyant, le paroleur nonpareil qui, dans Les Anthropoïdes, initie le jeune Guito aux mystères du Verbe et de l'Origine, trouve lui aussi un précurseur dans cet impayable Jodoin, détenteur profane d'une clef sacrée.

Le capharnaüm est ventre mais aussi caveau. L'air y est glacial, la pénombre et la poussière y règnent. Pour Jodoin, contrairement aux paroissiens de Saint-Joachin, l'endroit n'a rien d'une source de plaisir. C'est le lieu sinistre d'un traumatisme oublié, d'une terreur latente; le dépôt où gît le ferment de cette "vase alluviale psychique " que Marin agitera pendant tout un semestre, et où prend racine la "langue dans sa splendeur originaire" (Le Semestre) que les anthropoïdes auront pour mission d'explorer. Derrière la porte que déverrouille Jodoin, c'est l'espace des livres, de l'être interdit qui s'ouvre; celui que n'aura de cesse d'approfondir le futur Bessette dans son désir d'origine et de salut. L'espace des livres, c'est-à-dire à la fois le lieu d'une involution 
et celui de l'éventuelle survie. Sa carrière, c'est à l'intérieur de ce capharnaüm qu'elle prendra place désormais, réalisant symboliquement le fantasme utérin impossible à assouvir autrement.

Nor for every eye, annonce si bien le titre du livre en anglais.

C'est vous, les livres? demande le camionneur venu prendre livraison de la cargaison capharnaümesque. Et l'autre, sans se troubler:

- Je lui répondis que, en effet, les livres, c'était moi (p. 146).

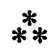

Outre la scène primitive décrite plus haut, je suis entré dans l'œuvre de Bessette par la porte souterraine, celle qui donne sur L'Incubation. Je venais d'arriver à l'université, je tombais simultanément sur Robbe-Grillet et Gilbert La Rocque, l'épopée de Gilgamesh et la psychanalyse de Marie Cardinal. J'ai lu le livre sans reprendre mon souffle, comme on fonce tête baissée dans un tunnel, emporté par un état d'euphorie et de frénésie qui, s'il faut en croire l'auteur, devait être assez proche de celui qui présida à l'écriture du texte lui-même (Les Dires d'Omer Marin). Je lisais trop vite pour pouvoir tout absorber, entraîné par le rythme seul, entièrement nouveau pour moi. Les mots retenaient toute mon attention au détriment de l'histoire, cette enfilade hypnotique de scènes heurtées, éthyliques. Londres sous les bombes, une auto, une route perdues̀ dans la nuit de l'Ontario. Un délire contrôlé, débité dans l'urgence la plus totale.

André Brochu a montré dans L'Instance critique la récurrence hallucinante des représentations du ventre dans ce roman où tout est boyau, avalement, digestion, y compris la phrase elle-même lorsqu'elle se ramifie et prolifère pour devenir ce labyrinthe au centre duquel on se retrouve à chaque ligne placé devant un nouveau dilemme, une fourchette de directions simultanées et quasi identiques, comme si la sélection paradigmatique n'opérait plus, laissant au lecteur le choix entre trancher lui-même ou se laisser circonvenir, assourdi, par un discours sans cesse court-circuité par l'accumulation des synonymes et paronymes. Le roman lui-même fonctionne à la façon d'un réseau organique qui se déploie pour engloutir le lecteur, un système sanguin à travers lequel l'œil et le cerveau effectuent une coupe en plongée, et dont la pulsation effrénée retentit à l'oreille, impossible à endiguer autrement que par ce retour au silence subit que signifie la mort. Bessette inaugurait ainsi une manière qui rompait définitivement avec son réalisme parfois laborieux du tournant des années soixante. 
Ce qui m'amène au fait que j'ai ensuite sauté dans Le Cycle à pieds joints, toujours séduit par ces phrases jamais terminées, toujours aussi attiré malgré moi par l'impossible nœud autour duquel le texte s'enroule sans jamais l'atteindre. J'y ai fait connaissance, au pied d'un cercueil encerclé par le clan familial, avec une obsession qui allait devenir familière au futur lecteur du Semestre: "[...] la chair les tissus l'ossature lentement implacablement rẹtournent. à l'indifférencié primordial" (p. 82).

Les deux pôles de l'œuvre sont, dans Le Cycle, clairement exposés: d'un côté, le tout-puissant patriarche transmetteur de semence dont la dominance sexuelle ou intellectuelle, sous les avatars du guerrier et du pédagogue, s'exerce tant à travers Les Antbropoïdes que dans Le Semestre; de l'autre, cet état indifférencié de la matière, propre à la terre femelle auquel tout vivant est promis, et dont se rapproche le père Barré couché dans sa boîte au milieu de sa progéniture. C'est vers ce même état indifférencié que tendent aussi, sous des déguisements variés à travers l'œuvre, "l'animalité la plus horizontale" et "la prime enfance immémorielle" (Le Semestre) de l'individu aussi bien que de la race. Entre le patriarche archaïque et sa progéniture symbolique ou non va se jouer et se rejouer le drame d'un "antique implacable œdipe antéconscienciel "( Le Cycle) dont l'enjeu ultime est le retour à l'humus-mémoire du géniteur archétypal et son remplacement par la génération suivante.

Bessette raconte dans Mes romans et moi que, l'automne, pour les besoins d'un jeu, il s'enfouissait sous un tas de feuilles mortes d'où il devait jaillir et attraper un des participants restés à former le cercle à l'extérieur. Le petit Bessette trouve alors intéressant de prolonger le plus possible son séjour souterrain:

[...] je me sentais à la fois enseveli et défunt en quelque sorte - mais capable de renaître à la volonté et de terroriser par ma seconde naissance les faibles créatures de l'extérieur.

Or, cette seconde naissance ne sera possible qu'à contre-temps: il faut enfiler la vie à l'envers, prendre comme point de départ sa propre mort. En voie d'être ravalé par le ventre du monde, l'antique géniteur ne peut trouver son salut que dans la parolade, c'est-à-dire le récit présenté, dans Les Anthropoïdes, comme voyage "à rebours dans le ventre du temps." 
Il s'agit sans doute d'une des tentatives romanesques les plus extravagantes de toute l'histoire de la littératuré québécoise. Le désir de régression semble y atteindre sa limite, servi par un langage que l'auteur a extrait de la tourbe mémoriale pour être donné aux premiers hommes. Langage élaboré dans sa structure, même s'il vise à reproduire la spontanéité du flux conscienciel brut, langage en partie inventé aussi, avec des ajouts lexicaux plutôt naifs, à mi-chemin entre le baby talk des amoureux et l'argot parisien. Exemples: Nanéa veut dire "femelle pubère", kroulou "vieillard", etc.

Sur le plan manifeste, le récit apparaît souvent comme simple prétexte à mettre en scène des fantasmes adolescents ou sadiques, teintés de cette misogynie si hargneuse qui, chez Bessette, ira en s'aggravant avec l'âge. La queue de ces guerriers Kalahoumes est aussi prompte à se dresser que leur casse-tête ne l'est à s'abattre et leurs odorantes femelles, qui forment au centre de la horde un noyau accessible aux seuls mâles dominants ("le noyau des femelles interdites"), sont pourvues de "vaste[s] cul[s] violet[s]", de "fentes dévoreuses", d' enfourchures insatiables" et de "longues crinières jumenteuses " qu'il s'agit pour l'homo plus ou moins sapiens d'empoigner de la plus classique manière, qui est la manière forte. La femme passe au cash. Les infidèles, celles qui ont cru pouvoir se refaire une vie hors de la tribu voire unir leur sort à un membre d'un groupement rival - se voient ramenées devant l'entrée de la caverne où on leur enfonce un épieu dans le vagin avant de les livrer encore vivantes à l'appétit des hyènes. Bien sûr, le contexte préhistorique donne beau jeu à l'auteur. Mais, d'autre part, n'étaient la complexité formelle et la salacité féroce des étreintes, on pourrait être tenté de voir dans ce livre un ouvrage destiné à la jeunesse, étiquette avec laquelle Bessette lui-même paraît flirter quand il annonce en page couverture, avec la complicité de son éditeur, un roman d'aventure(s). Aventures, donc, de ces hominiens exemplaires dont l'auteur traduit pour notre bénéfice les grognements gutturaux et inauguraux, véritable babil de l'humanité, et qui, à travers leurs guerres d'escarmouches et leurs rapines, sont déjà aux prises avec la quête du Sens. Aventure, aussi, du langage lui-même, puisque, quelques millénaires avant l'apparition des rhéteurs grecs, la forme à donner à la parolade archaïque se révèle déjà aussi importante que l'histoire qu'elle veut raconter. "[...] le paroleur parolant à la horde ne doit pas parler de lui-même sauf à l'orée de son discours" (p. 4). "[...] le bon paroleur n'est jamais seulement lui-même et (il) n'est jamais seulement en lui-même" (p. 5).

Pour cette humanité perçue dans son enfance, la blessure du cordon ombilical est encore toute fraîche. Le ventre, lieu des plaisirs et 
des servitudes, de la survie et de la perpétuation de l'espèce, se trouve partout. Comme une aile protectrice, il recouvre ce noyau d'humanité de son ombre inaugurale, il est la grotte qui accueille le bipède dans les profondeurs de la terre. Le temps a un ventre. Le récit a un ventre. Espace idéalisé, voué aux séductions de l'origine comme à la perspective commune du retour à l'indifférence, le ventre devient accoucheur de possible. Refuge du refoulement absolu, il retourne le temps, enfante le récit.

Si Bessette répond à ses propres questions dans Les Anthropoïdes, c'est en remontant à la source logique d'un constat qui finit par former l'assise même de sa pensée: le primat, dans toutes les phases de l'existence, de notre nature physiologique et de notre animalité foncière, unique et pauvre vérité qui fait de nous des êtres soumis aux aléas de la vie organique et de ses cycles et nous condamne à nous rapprocher périodiquement de l'horizontale avant de nous y écraser pour de bon, en une heureuse et définitive dissolution de l'ego. Nulle transcendance n'est possible sinon dans la parole, qui elle-même naît du chaos des instincts. Nos pensées les plus intimes peuvent être retracées scientifiquement. Un double déterminisme, psychique et génétique, s'ajoute chez Bessette à l'idée de contingence pour engendrer un compromis instable, sorte de cul-de-sac gnoséologique où la résignation n'abolit pas l'inquiétude, et au fond duquel l'individu se débat aussi seul, anonyme et transitoire que nécessaire et complexe. L'être humain est le produit d'une rencontre accidentelle et personne ne peut revenir en arrière, mais dans le secret de la psyché, il est permis de se retourner pour contempler, effrayant de familiarité, ce ventre maternel aveugle où le Cosmos a planté sa mise. Observant les vagues du lac Ontario, Omer Marin se laisse aller à rêvasser devant ces "millions de crêtes déferlantes aussi insignifiantes-contingentes (ou - ce qui (revient) au même - aussi inéluctables-déterminées) que les milliards de fotus (de l'bomo censément sapiens). (Le Semestre). Bessette, on le voit, c'est la contingence sans la liberté au bout du tunnel, la vie humaine comme donnée improbable qui, dès le moment de la naissance, dégénère en un processus pour ainsi dire déjà accompli. Le hasard fait place au pattern. Aussi, quand la matrice est le site principal où doit se décider l'alchimie, comment ne pas regretter les délices qu'on y goûtait, ce perpétuel devenir, cette pure potentialité de soi-même? Rester en attente des choses, bien au chaud et intact, éternellement suspendu au seuil de la dégringolade qui va faire du corps un objet à dégrader.

Considérer la vie comme un gaspillage de forces inutiles, c'était déjà le point de vue de Jodoin: l'individu s'entoure d'un carcan 
d'habitudes, s'invente une sécurité en accord avec la loi de la dépense minimale. L'horreur des déplacements lui tient lieu de principe moral. "Sur le chapitre de la digestion, observe pour sa part Chayer, le narrateur de La Commensale, je ne transige pas.. (p. 13)

L'écrivain Bessette, assis entre Freud et Darwin, ne manque pas de pères substituts pour occuper la place laissée vacante par l'Église. Il lui reste encore à s'élever lui-même à ce rang. L'“antique pédagogue un peu toqué " (Le Semestre) ne sera que l'autre versant de l'anthropoïde brutal et sanguinaire qui hante l'œuvre et fait peser sur tout l'édifice bessettien, y compris ses acquis les plus civilisés (la psychocritique, la pratique de la littérature), la menace permanente d'une "irréversible involution" (Ibid.). C'est à se demander jusqu'où l'écriture peut pousser un homme, jusqu'à quelles hauteurs elle doit le hisser avant de pouvoir le précipiter de nouveau dans sa condition périssable. Quelle intervention miraculeuse faut-il espérer chez celui qui se sait écrit par tous les autres?

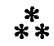

Je n'ai jamais été très attiré par les livres dits réalistes de Bessette. Les interminables dialogues de La Bagarre me rebutent, et Les Pédagogues dorment bien tranquilles sur une étagère de ma bibliothèque. L'écriture de ces deux livres me paraît trop bêtement appliquée; je préfère, sans doute par masochisme, les pages où Bessette ouvre vraiment les vannes pour laisser libre cours à sa libido exaspérée et aux raffinements maniaques de son travail subconscient.

Le Semestre s'est retrouvé un beau jour, ou une bonne nuit, à l'affût sur ma table de chevet. J'ai parcouru ce livre comme on traverse une vie entière, avec tout ce que ça peut charrier d'amertume, de retours en arrière pénibles et de petits plaisirs malins en forme d'oubli, disséminés au milieu d'une intolérable mer de lucidité. Dans mon texte sur Bessette, je voulais parler surtout du Semestre, livrephare d'un homme seul, ou livre-clef de voûte d'une vie fictive, comme on voudra. Je me rends compte que l'espace va me manquer, et que c'est peut-être mieux ainsi. Ce livre décourage le commentaire à force d'aller au-devant, à force de se regarder s'auto-engendrer, entre néant et nombril. C'est un exercice de lecture dangereux, chaque page étant piégée, chaque phrase susceptible à tout moment de se retourner sur elle-même pour montrer son envers, ou son vide. L'homme-vieillissant-écrivain-en-exil-enseignant-sur-le-retour que vous 
commenciez à pouvoir haïr sans retenue, en le taxant d'égotisme aigu ou de narcissisme rédhibitoire et de bien d'autres maux pour la définition desquels il se montre toujours prêt à vous offrir de sa propre initiative un flanc généreux, cet homme vous revient sans prévenir, le paragraphe d'après, avec une phrase comme celle-ci: "s'il n'y avait pas la souffrance, comme il serait facile de se ficher de tout "(p. 175).

Vous aimeriez être capable de vous offusquer de ses jugements esthétiques péremptoires et grinçants, souvent prétentieux, des règlements de compte qu'il expédie à l'emporte-pièce mine de rien et des incessantes attaques qu'il mène contre tout ce qui grouille à l'époque dans le paysage littéraire québécois et ne s'appelle pas Gilbert La Rocque, ci-devant directeur littéraire de̊s éditions Québec/Amérique et responsable, à ce titre, de la publication du Semestre. Mais Bessette arrive à vous faire partager sa joie mauvaise, sa mesquine jubilation. Une main devant la bouche, vous riez de votre propre amusement, vous trouvez puéril et donc inoffensif de débusquer toute une galerie de gendelettres derrière les transparents masques dont les affuble la fausse pudeur de l'auteur: les Butor-Ali Nonlieu, Jean Lasile, Chienlit Piton, Naif Quatrânes, Tonton Micon, Jean-Achier Laid et compagnie... Pardon, pardon d'avoir pris plaisir à ces impertinences.

$\mathrm{Au}$ moment où enfin vous vous sentez prêt à rembarrer, en refermant le livre, ce singe mis à nu, si maniéré et qui s'estime, sans doute par excès de modestie, surdéveloppé du cerveau, le voilà qui se met à rire paisiblement de lui-même, qui agite sous vos yeux ses jouets dérisoires et pose sur son personnage un regard dépourvu de complaisance, se moquant avec vous de cet "indigeste jargonnage" (p. 127) dont il est le dernier dupe, de sa "logorrhée-graphorrée" (p. 11) en apparence ausssi irrépressible que le mouvement premier de sa pensée, et qu'il affirme, honnête provocateur, ne pas savoir distinguer de la bonne littérature. Dans les méninges de ce puissant "jongleur-marchand de mots" (p. 25), de cette "médiocre bête pédagogique" (p. 24) et de ce "bizarre anachorète involutif " (p. 273) qui est aussi un " chercheur d'or lunatique" (p. 133) et un chasseur-rêveur en cage, persiste malgré tout le fantasme d'une littérature qui serait pure, débarrassée des convenances, non apprivoisée, une littérature qui serait "un dire irrésistible ailleurs que dans la préhistoire" (p. 103).

Le Semestre, ou l'apothéose du roman de prof. À la question hypothétique: Que peut-on savoir d'un bomme aujourd'bui?, Bessette semble faire le pari d'une réponse qui ne se peut qu'au prix d'un égocentrisme vertigineux, d'une volonté d'introspection qui atteint ici sa phase terminale, comme l'implosion d'une passion. Dans la scène 
originaire de Serge d'entre les morts, que le professeur Marin tire du roman de La Rocque pour la mettre au centre du sien, tous les éléments sont en place pour amener l'entreprise romanesque à se résorber, la parole autoritaire à se taire. Se fantasmant tour à tour comme père devant l'étudiante Sandra, comme fils à travers le La Rocque enfant, et encore une fois comme père (supposé) d'un rejeton qu'il se (re)découvre au terme d'un travail d'analyse acharné, Marin peut enfin, porté par la logique implacable de la scène primitive, se voir raide mort comme à travers des yeux d'enfant. Ce n'est pas un hasard si, dans Les Dires d'Omer Marin qui seront son dernier livre, oublié, Bessette prête cette fois la narration à un ancien élève de Marin, victime lui aussi du maudit CEdipe universitaire. Comme si la boucle avait été bouclée, et bien bouclée, dans les enroulements successifs étourdissants de la scène originaire qui aboutissent dans Serge d'entre les morts à la désintégration de la figure patriarcale: l'archaïque porteur de semence, effacé par une tempête de neige, effectue son retour à l'opaque du monde à travers une poudrerie comme on n'en avait pas vue depuis le jour de la naissance d'un certain Gérard Bessette, dans le ventre de l'hiver 1920 .

\section{$\stackrel{*}{* *}$}

Il paraît qu'on peut encore l'apercevoir parfois, antique pédagogue blanchi sous le harnais académique, "homo (à demi) ontariensis" errant de par cette Narcotown soporifique et périphérique qui lui a apporté, entre autres bienfaits, la paix nécessaire à la production d'une ouvre. Le fantôme de Gérard Bessette continue de hanter les rives du lac Ontario et les parcs de Kingston aux ormes morts, posant à des passantes imaginaires, filles bien roulées pour la plupart, cette question sans réponse, ni repos:

Tous ces fantasmes et cette manie scripturale lui avaient-ils donné une vie une vraie vie? 